\title{
Rede Nordeste de Biotecnologia (RENORBIO): um panorama atual do programa
}

\author{
Northeast Biotechnology Network (RENORBIO): a current overview of the \\ program

\section{Connexion du Nord-Est en Biotechnologie (RENORBIO): une vue d'ensemble en cours du programme}

\author{
Red de la Biotecnología del Noreste (RENORBIO): una visión actual del \\ programa
}

\author{
Mário Luan Silva de Medeiros ${ }^{1}$ \\ Josimara Nolasco Rondon ${ }^{2}$
}

\begin{abstract}
Recebido em 24/06/2016; revisado e aprovado em 23/05/2017; aceito em 23/05/2017
\end{abstract}
DOI: http://dx.doi.org/10.20435/inter.v19i1.1124

\begin{abstract}
Resumo: O estudo teve como objetivo elucidar o panorama evolutivo da Rede Nordeste de Biotecnologia (RENORBIO), desde a sua criação em 2006 até o período de 2015. Para tanto, utilizou-se como metodologia a pesquisa quantitativa e os métodos investigativo exploratório e descritivo. O estudo mostrou que a RENORBIO é composta por 35 instituições de ensino e pesquisa, por mais de 172 docentes/pesquisadores, já formou 361 doutores e possui mais de 140 depósitos de patentes.
\end{abstract}

Palavras-chave: Renorbio; pós-graduação em Biotecnologia; nordeste brasileiro.

Abstract: The study aimed to elucidate the evolutionary panorama of the Northeast Biotechnology Network (RENORBIO), since its inception in 2006 to the period of 2015. Therefore, it was used as a methodology to quantitative research and exploratory and descriptive investigative methods. The study showed that RENORBIO consists of 35 teaching and research institutions, more than 172 teachers/researchers, has trained 361 doctors and has over 140 patent applications.

Keywords: Renorbio; Biotechnology post-graduation; brazilian northeast.

Résumé: L'étude visait à élucider le panorama évolutif de la biotechnologie Network Northeast (RENORBIO), depuis sa création en 2006 à la période de 2015. Par conséquent, il a été utilisé comme une méthode de recherche quantitative et des méthodes d'investigation exploratoires et descriptives. L'étude a montré que RENORBIO se compose de 35 enseignement et de recherche, plus de 172 enseignants/chercheurs, a formé 361 doctors et compte plus de 140 demandes de brevet.

Mots-clés: Renorbio; post-diplômé en Biotechnologie; nord-est du Brésil.

Resumen: El estudio tuvo como objetivo aclarar el panorama evolutivo de la Red de Biotecnología Noreste (RENORBIO), desde su creación en 2006 hasta el período de 2015. Por lo tanto, se utilizó como metodología de investigación cuantitativa y métodos de investigación exploratoria y descriptiva. El estudio mostró que RENORBIO consta de 35 instituciones de enseñanza e investigación, más de 172 profesores/investigadores, ha capacitado a 361 doctores y tiene más de 140 solicitudes de patentes.

Palabras clave: Renorbio; postgrado en Biotecnología; nordeste brasileño.

\section{INTRODUÇÃO}

Este estudo apresenta o panorama atual do Programa de Pós-Graduação da Rede Nordeste de Biotecnologia (RENORBIO). Os dados explorados evidenciam a evolução da RENORBIO desde a sua inserção no âmbito científico pela Coordenação de Aperfeiçoamento de Pessoal de Nível Superior (CAPES) em 2006, até o período de 2015. Dessa forma, pelo importante acréscimo

\footnotetext{
${ }^{1}$ Universidade do Estado do Rio Grande do Norte (UERN), Mossoró, Rio Grande do Norte, Brasil.

${ }^{2}$ Universidade Católica Dom Bosco (UCDB), Campo Grande, Mato Grosso do Sul, Brasil.
} 
econômico e ainda pelo quantitativo de pesquisadores formados na Região Nordeste, os dados apresentados neste documento acrescentam informações relevantes acerca de como a RENORBIO vem contribuindo para o crescimento da pesquisa no campo científico da Biotecnologia.

A RENORBIO se concretizou como fruto de excelência perspectivando potencializar a pesquisa, desenvolvimento e inovação - PD\&I da Biotecnologia do nordeste brasileiro (COSTA, 2012). Esse incremento se institucionalizou pelo Ministério da Ciência e Tecnologia (MCT), via Portaria MCT n. 598, publicada no Diário Oficial em 30 de novembro de 2004 (DOU de 30.11.2004, Seção I, p. 16) (QUINTELLA et al., 2013).

O nordeste brasileiro, a partir da criação da RENORBIO, teve a oportunidade de desenvolver núcleos de pesquisa de biotecnologia, favorecendo e engrandecendo o mercado empresarial e industrial, como também os aspectos de pesquisa em unidades de ensino superior (RENORBIO, [s.d.]a).

Assim sendo, como forma de adicionar contribuições ao tema, a presente investigação teve como objetivo geral elucidar o panorama evolutivo da RENORBIO. Para tanto, utilizou como metodologia a pesquisa quantitativa e os métodos investigativos exploratório e descritivo. Os dados apresentados neste estudo foram coletados através de informações bibliográficas disponibilizadas sobre a RENORBIO no site do Programa e ainda em bancos de dados da Coordenação de Aperfeiçoamento de Pessoal de Nível Superior (CAPES), da Plataforma Sucupira, do Conselho Nacional de Desenvolvimento Científico e Tecnológico (CNPq) e na Biblioteca Digital Brasileira de Teses e Dissertações (BDTD).

Posto isso, o texto ora apresentado encontra-se organizado em quatro momentos. No inicial, discorreu-se a respeito dos procedimentos metodológicos deste estudo. No segundo instante, foram expostos os aspectos gerais da RENORBIO. Em sequência, descreveu-se a organização do programa e, no último momento, versou-se acerca dos principais aspectos constituídos no período de 2006 a 2015 da RENORBIO.

\section{PROCEDIMENTOS METODOLÓGICOS DO ESTUDO}

O estudo em questão trata de uma pesquisa quantitativa com a utilização do método exploratório e descritivo, o qual evidencia o panorama atual do Programa de Pós-Graduação da RENORBIO.

A bibliografia disponível sobre o tema foi a fonte de coleta dos dados, como também as principais referências de informações para a presente pesquisa. Acredita-se que o estudo exploratório e descritivo deve desenvolver-se de fontes diversas. A bibliografia acerca de um tema é um dos meios em que os pesquisadores das Ciências Naturais têm para ampliar o entendimento do que se pesquisa e utilizar desse instrumento para sintetizar aquilo que é investigado por outros investigadores ou sistematizar os conhecimentos existentes (GIL, 2008).

Nessa perspectiva, utilizou-se como fonte principal de dados, as informações bibliográficas disponibilizadas na página do site do Programa de Pós-Graduação RENORBIO, como também fez-se uso de informações coletadas em documentos acerca da RENORBIO, mais especificamente dos dados referentes ao programa, tais como: o Regimento Geral do Programa de Pós-Graduação da RENORBIO, o relatório de produtos e processos desenvolvidos nas pesquisas de doutoramento pela RENORBIO e uma tese que aborda a expansão de Redes em Biotecnologia no nordeste brasileiro ${ }^{3}$.

\footnotetext{
${ }^{3}$ Da Bancada ao Bureau: análise do desenvolvimento da biotecnologia no Nordeste Brasileiro sob enfoque do Sistema Regional de Inovação. Tese de autoria de Benedita Marta Gomes Costa, desenvolvida na pós-graduação em Biotecnologia pela RENORBIO no ano de 2012.
} 
Reforça-se que, tencionando concretizar o objetivo central desse estudo, fez-se uso ainda do banco de teses da CAPES (e ainda o Portal de Periódicos CAPES/MEC), da Plataforma Sucupira, do diretório de pesquisa do CNPq e da BDTD.

Foi a partir dessas fontes que se realizou o levantamento sobre os dados de relevância (no período de novembro de 2015 a janeiro de 2016) para dar suporte ao conhecimento do panorama atual do programa RENORBIO. Inicialmente, buscaram-se os dados concernentes às principais universidades da Região Nordeste que participam da RENORBIO, conhecidas na literatura nacional como pontos focais; o número de docentes/orientadores permanentes ao programa por ponto focal ${ }^{4}$; as áreas de concentração/linhas de pesquisa, acatadas pelo programa, atentando-se às principais áreas entre o período de 2006 a 2015; como também a quantidade de discentes ingressantes durante o período eloquente.

Por seguinte, buscaram-se os principais produtos e processos formulados através das pesquisas desenvolvidas pela RENORBIO disponibilizados em documento para consulta no site da RENORBIO, sendo categorizados pelas áreas de aplicação.

Em continuidade, verificou-se o total de teses publicadas e disponibilizadas durante o período de 2006 a 2015 pela RENORBIO. Esse procedimento foi realizado no banco de teses da CAPES, no qual se inseriu e se considerou para a busca o termo RENORBIO (no setor de programas de pós-graduação) e o ano de defesa da tese; como na Plataforma Sucupira, considerando-se o número de teses defendidas por ponto focal.

No Portal de Periódicos CAPES/MEC, em sequência, também considerou-se o termo RENORBIO, para a busca de artigos sobre o tema (como tipo de material), entre o período de 2006 a 2015. Esta foi realizado na BDTD, em busca avançada de teses utilizando o termo RENORBIO (todos os campos), entre o período de 2006 a 2015.

No último momento de coleta de dados, verificaram-se os grupos de pesquisa cadastrados no diretório de pesquisa do CNPq na Região Nordeste, articulando com as unidades de pontos focais da RENORBIO.

Após esses procedimentos, organizaram-se os dados coletados em gráficos, quadros e tabela, para posteriormente desenvolver a análise quantitativa do material e o relatório com os resultados da investigação apresentado neste documento.

\section{RENORBIO: ASPECTOS GERAIS}

A RENORBIO foi criada a partir de um protocolo entre o Instituto Nacional do Semiárido (INSA) e o Ministério da Ciência e Tecnologia (MCT), em 2004, com a finalidade de incentivar o processo de contribuição na Região Nordeste com tecnologia e indústria, tendo inicialmente, como linhas de pesquisa, setores voltados para a bioprospecção de plantas com propriedades medicinais e/ou funcional aos seres humanos, a otimização de processos e/ou produtos biotecnológicos voltados como ferramentas para os seres humanos (vacinas, kits de diagnósticos, terapias), o estudo genético de sistemas de beneficiamento vegetal e a inovação de técnicas, e/ ou criação, aplicadas à reprodução animal (COSTA, 2012).

Assim, as necessidades de formação de corpo técnico em Biotecnologia e da divulgação do conhecimento produzido pelos grupos de pesquisa em tecnologia, observados a partir do

\footnotetext{
${ }^{4}$ Ponto focal refere-se às principais universidades participantes da RENORBIO, como apoio científico/pedagógico para os discentes e pesquisadores.
} 
Fórum da Comunidade em Biotecnologia em 2004, foram fatores motivadores para o processo de criação de um programa de pós-graduação em rede e em Biotecnologia, no âmbito da Rede Nordeste de Biotecnologia (QUINTELLA et al., 2013).

Dessa forma, no mesmo ano, foi lançado um edital (Etene/FUNDECI/MCT) ${ }^{5}$ para financiamento de projetos relacionados às áreas de pesquisa acima mencionadas, com a finalidade de se verificar como os projetos iriam se agrupar na formação de redes de pós-graduação, na execução das atividades (COSTA, 2012).

As instituições contempladas foram a Universidade Federal de Pernambuco (UFPE), a Universidade Federal do Rio Grande do Norte (UFRN), a Universidade Estadual do Ceará (UECE) e o Centro de Pesquisa Gonçalo Muniz (CPpGM). Consolidado em 2006, pela CAPES, o Núcleo de Pós-Graduação da Rede Nordeste de Biotecnologia foi a primeira proposta de porte regional aprovada pela CAPES na área de Biotecnologia na Região Nordeste (RENORBIO, [s.d.]c).

O Programa de Pós-Graduação em Biotecnologia da RENORBIO iniciou suas atividades em 2006, ofertando um Curso de Biotecnologia na modalidade de doutorado, destinado à formação de docentes e pesquisadores no campo de Biotecnologia (BRASIL, s.d.).

Constituído inicialmente por 28 instituições de ensino e pesquisa (atualmente 35), a RENORBIO possui como objetivos centrais a formação de pessoal qualificado para o exercício da pesquisa e do magistério superior, considerados indissociáveis no campo da Biotecnologia, o incentivo à pesquisa na área de Biotecnologia, sob perspectiva multi e interdisciplinar e a produção, difusão e aplicação do conhecimento da Biotecnologia na realidade econômica e cultural da Região Nordeste (BRASIL, s.d.).

O programa possui áreas de concentração estabelecidas em saúde, agropecuária, recursos naturais e biotecnologia industrial, sendo a sede administrativa do programa localizada em endereço itinerante, definida a cada três anos, de acordo com a localização da coordenação geral, pelo colegiado geral, possuindo como metas a construção de um centro de excelência virtual em biotecnologia que, por sua vez, se focaliza na indústria (COSTA, 2012; RENORBIO, [s.d.]c). A figura 1 ilustra essas considerações:

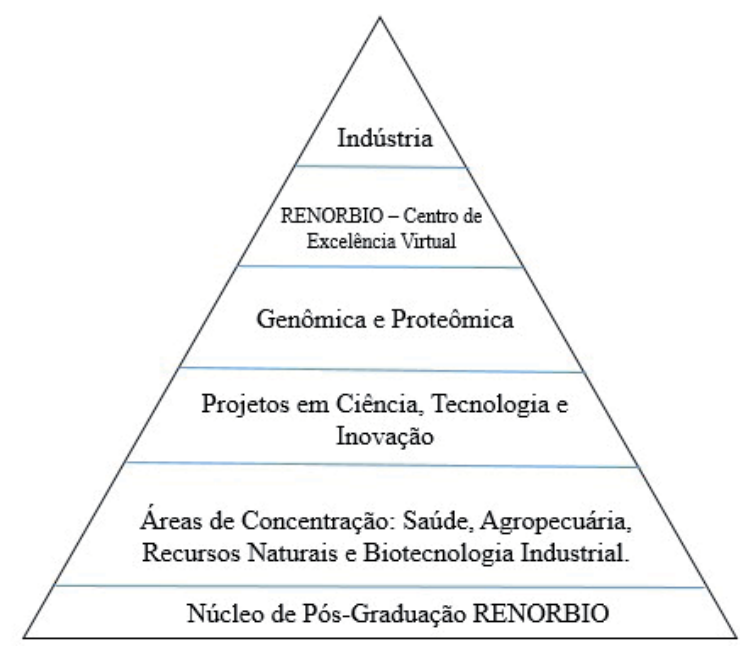

Figura 1 - Organização das principais metas da Rede Nordeste de Biotecnologia (RENORBIO)

Fonte: Adaptado de Costa (2012)

\footnotetext{
${ }^{5}$ Escritório Técnico de Estudos Econômicos do Nordeste e Fundo de Desenvolvimento Científico e Tecnológico.
} 
Assim sendo, em longo prazo "espera-se que a RENORBIO se constitua em um Núcleo de Excelência em Biotecnologia, que internalizará e desenvolverá as tecnologias mais avançadas para aplicação ampla em todas as áreas da Biotecnologia, com adequados níveis de excelência e relevância" (RENORBIO, [s.d.]b), aumentando o fluxo de inovação e beneficiando a sociedade com os seus produtos e serviços.

A exemplificar, já foram formulados produtos de cunho tecnológico e de inovação como, "Fungicida natural, eficaz no combate às pragas de fungos resistentes, com baixo custo de produção" (RENORBIO, [s.d.]f, p. 08), "Fitoterápico com atividade antiinflamatória e ação antimicrobiana para bactérias e leveduras orais. Eficaz no controle de inflamações e do crescimento microbiano da cavidade oral" (RENORBIO, [s.d.]f, p. 18), "Produção de biopapel a partir da associação de microorganismo" (RENORBIO, [s.d.]f, p. 64), e "Desenvolvimento de rações para ruminantes obtidas a partir de plantas nativas com ação anti-helmíntica de baixa toxicidade, menor período de carência para o consumo de produtos de origem animal, e baixo poder de seleção de parasitas resistentes" (RENORBIO, [s.d.]f, p. 108).

\section{NÚCLEO DE PÓS-GRADUAÇÃO}

O Núcleo de Pós-Graduação (NPG) da RENORBIO tem caráter multi-institucional, para adesões é necessária uma aprovação pela instituição do regimento do NPG definida na Portaria MCT n. 598, de 26 de novembro de 2004. O excerto textual a seguir, elucida entendimentos:

Essa Portaria define que a integração ao NPG do RENORBIO, como membro efetivo, requer que o pesquisador esteja ativamente envolvido nos projetos da Rede. O Regimento, formalizado por Protocolo de Intenções firmado pelos dirigentes institucionais que concordaram em aderir à proposta do Núcleo de Pós-Graduação do RENORBIO, explicita que essa adesão implica a participação de seus docentes na orientação de alunos em projetos de pesquisa e de teses; na oferta, pelos docentes da instituição, de disciplinas que compõem o currículo do curso para os alunos que integrarão o Programa de Pós-Graduação em Biotecnologia, bem como a utilização da infra-estrutura de ensino e pesquisa instituição para atender aos objetivos do Programa. 0 intercâmbio de docentes e discentes também é igualmente fomentado, bem como a elaboração e execução de projetos de pesquisa em rede, visando ao estabelecimento de plataformas de competência em projetos acadêmicos e tecnológicos de interesse comum. (RENORBIO, [s.d.]c).

Atualmente, esse Núcleo de Pós-Graduação (NPG) da RENORBIO é coordenado pela Professora Doutora Maria Madalena Pessoa Guerra, da Universidade Federal Rural de Pernambuco (UFRPE), tendo como vice-coordenadora a Professora Doutora Aurea Wischral, da mesma instituição, havendo ainda um representante estadual, coordenador e vice-coordenador, pertencente ao ponto focal daquela região.

Além desses membros de excelência no âmbito estadual e coordenador e vice-coordenador geral, há membros representantes para as áreas de concentração por Estado. Esse colegiado é responsável pelas atribuições relevantes às áreas de concentração do programa, sendo formado por câmaras de biotecnologia em saúde, de biotecnologia em recursos naturais, de biotecnologia industrial e de biotecnologia em agropecuária (RENORBIO,[s.d.]c). Atualmente, além dessas áreas de concentração, há a área de concentração denominada de bionegócios e marcos legais (RENORBIO, [s.d.]h).

Dessa forma, o NPG da RENORBIO encontra-se organizado da seguinte maneira: coordenador geral, vice-coordenador geral, coordenador executivo da RENORBIO, representantes estaduais, coordenadores das câmaras, representes estudantis e o secretário executivo (BRASIL, s.d. ). 
Com o objetivo geral de formar profissionais no âmbito da pesquisa e ensino, como também na prestação de serviços e indústrias, o NPG da RENORBIO, almeja contribuir no processo de consolidação/continuação do programa de pós-graduação da RENORBIO, como também no desenvolvimento da Biotecnologia no Brasil (RENORBIO, [s.d.]c).

Para tal efeito, o NPG conta com uma grade estrutural composta de mais de 160 laboratórios de pesquisa, distribuídos pelas instituições a eles ligadas, também com espaços para docentes e discentes, como bibliotecas e laboratórios de informática (RENORBIO, [s.d.]g).

Nessa conjuntura, o público-alvo do programa é formado por professores universitários e profissionais de nível superior interligados às áreas de Biotecnologia-saúde, recursos naturais, indústria e agropecuária (RENORBIO, [s.d.]c).

A seleção se iniciou de forma anual por meio de prova de conhecimentos em duas línguas estrangeiras, seguida por defesa de anteprojeto, entrevista e análise do Curriculum Vitae (BRASIL, s.d.). Atualmente, a seleção é composta por avaliação do projeto de pesquisa on-line (eliminatório) e análise do curriculum vitae (eliminatório e classificatório), em que se deve considerar a área de concentração de interesse, o ponto focal e o orientador.

Para se ter uma ideia, no período de 2006 a 2015, já houve mais de 11 seleções, com uma média de inscritos de 200 por seleção (RENORBIO, [s.d.]i).

O curso de pós-graduação da RENORBIO tem duração mínima de 24 meses e duração máxima de 48 meses, com a matriz curricular organizada de acordo com a área de concentração pela qual o participante optou no ato da seleção, totalizando 48 créditos -14 créditos de disciplinas obrigatórias, 14 créditos de disciplinas eletivas, 16 créditos da tese e quatro créditos do estágio à docência (BRASIL, [s.d.]; RENORBIO, [s.d.]c).

Por ser o primeiro programa de pós-graduação em Rede em nível de doutoramento no Nordeste Brasileiro, a RENORBIO apresentou-se como forte candidata, devido ao suporte científico e técnico que o foi desafiado em todos os Estados do Nordeste, com uma inter-relação de distribuição de conhecimento científico e de suporte técnico para os discentes, facilitando o desenvolvimento dos trabalhos de doutoramento e se diferenciando dos demais programas distribuídos pelo País, por ter essa inter-relação menos abrasiva e menos burocrática, como também a diversidade de áreas de concentração.

\subsection{Pontos focais e corpo docente /provedores do saber}

A RENORBIO compõe-se atualmente de 35 instituições de ensino e centros de pesquisa distribuídos pelo Nordeste Brasileiro, divididos por pontos focais (RENORBIO, [s.d.]d). Esses pontos focais, no total de 12, são considerados como pontos de acesso à RENORBIO e responsáveis pelo gerenciamento da rede naquela região (RENORBIO, [s.d.]e).

O quadro 1 alude a todas as instituições e centros de pesquisa da RENORBIO, distribuídos por pontos focais. Essa demanda de instituições e centros de pesquisa por pontos focais é realizada pela conveniência do instituto com o ponto focal por Estado. 


\begin{tabular}{|c|c|c|c|}
\hline ESTADO & PONTOS FOCAIS & INSTITUIÇÕES E CENTROS PARTICIPANTES & REPRESENTANTE ESTADUAL \\
\hline \multirow{2}{*}{ Espírito Santo (ES) } & \multirow{2}{*}{$\begin{array}{l}\text { Universidade Federal } \\
\text { do Espirito Santo } \\
\text { (UFES) }\end{array}$} & $\begin{array}{l}\text { Instituto Capixaba de Pesquisa e Extensão } \\
\text { Rural (INCAPER) }\end{array}$ & \multirow{2}{*}{$\begin{array}{l}\text { Prof. Dr. Antônio Alberto } \\
\text { Ribeiro Fernandes - UFES }\end{array}$} \\
\hline & & Universidade Federal do Espírito Santo (UFES) & \\
\hline \multirow{2}{*}{ Maranhão (MA) } & \multirow{2}{*}{$\begin{array}{l}\text { Universidade Federal } \\
\text { do Maranhão (UFMA) }\end{array}$} & Universidade Estadual do Maranhão (UEMA) & \multirow{2}{*}{$\begin{array}{l}\text { Prof. Dr. Lívio Martins Costa } \\
\text { Júnior - UFMA }\end{array}$} \\
\hline & & Universidade Federal do Maranhão (UFMA) & \\
\hline \multirow{3}{*}{ Piauí (PI) } & \multirow{3}{*}{$\begin{array}{l}\text { Universidade Federal } \\
\text { do Piauí (UFPI) }\end{array}$} & Embrapa Meio-Norte (CPAMN) & \multirow{3}{*}{$\begin{array}{l}\text { Profa. Dra. Maria Acelina } \\
\text { Martins de Carvalho - UFPI }\end{array}$} \\
\hline & & Universidade Estadual do Piauí (UESPI) & \\
\hline & & Universidade Federal do Piauí (UFPI) & \\
\hline \multirow{4}{*}{$\begin{array}{l}\text { Rio Grande do } \\
\text { Norte (RN) }\end{array}$} & \multirow{4}{*}{$\begin{array}{l}\text { Universidade Federal } \\
\text { do Rio Grande do } \\
\text { Norte (UFRN) }\end{array}$} & $\begin{array}{l}\text { Universidade Federal Rural do Semi-Árido } \\
\text { (UFERSA) }\end{array}$ & \multirow{4}{*}{$\begin{array}{l}\text { Profa. Dra. Lucymara } \\
\text { Fassarella Agnez Lima - } \\
\text { UFRN }\end{array}$} \\
\hline & & $\begin{array}{l}\text { Universidade Federal do Rio Grande do Norte } \\
\text { (UFRN) }\end{array}$ & \\
\hline & & Universidade Potiguar (UnP) & \\
\hline & & $\begin{array}{l}\text { Universidade do Estado do Rio Grande do } \\
\text { Norte (UERN) }\end{array}$ & \\
\hline \multirow[t]{2}{*}{ Alagoas (AL) } & \multirow{2}{*}{$\begin{array}{l}\text { Universidade Federal } \\
\text { de Alagoas (UFAL) }\end{array}$} & $\begin{array}{l}\text { Universidade Estadual de Ciências da Saúde } \\
\text { de Alagoas (UNCISAL) }\end{array}$ & \multirow{2}{*}{$\begin{array}{l}\text { Prof. Dr. Antonio Euzébio } \\
\text { Goulart Santana - UFPI }\end{array}$} \\
\hline & & Universidade Federal de Alagoas (UFAL) & \\
\hline \multirow{7}{*}{ Pernambuco (PE) } & \multirow{7}{*}{$\begin{array}{l}\text { Universidade Federal } \\
\text { Rural de Pernambuco } \\
\text { (UFRPE) e } \\
\text { Universidade Federal } \\
\text { de Pernambuco } \\
\text { (UFPE)* }\end{array}$} & Universidade Federal de Pernambuco (UFPE) & \multirow{7}{*}{$\begin{array}{l}\text { Profa. Dra. Maria Madalena } \\
\text { Pessoa Guerras - UFRPE e } \\
\text { Profa. Dra. Teresinha } \\
\text { Gonçalves da Silva - UFPI }\end{array}$} \\
\hline & & $\begin{array}{l}\text { Universidade Católica de Pernambuco } \\
\text { (UNICAP) }\end{array}$ & \\
\hline & & $\begin{array}{l}\text { Empresa Pernambucana de Pesquisa } \\
\text { Agropecuária (IPA) }\end{array}$ & \\
\hline & & $\begin{array}{l}\text { Centro de Pesquisa Aggeu Magalhães } \\
\text { (CpqAM) }\end{array}$ & \\
\hline & & $\begin{array}{l}\text { Universidade Federal Rural de Pernambuco } \\
\text { (UFRPE) }\end{array}$ & \\
\hline & & $\begin{array}{l}\text { Universidade Federal do Vale do São Francisco } \\
\text { (UNIVASF) }\end{array}$ & \\
\hline & & Universidade de Pernambuco (UPE) & \\
\hline \multirow{3}{*}{ Sergipe (SE) } & \multirow{3}{*}{$\begin{array}{l}\text { Universidade Federal } \\
\text { de Sergipe (UFS) }\end{array}$} & Embrapa Tabuleiros Costeiros (CPATC) & \multirow{3}{*}{$\begin{array}{l}\text { Prof. Dr. Charles dos Santos } \\
\text { Estevam - UFS }\end{array}$} \\
\hline & & Universidade Federal de Sergipe (UFS) & \\
\hline & & Universidade Tiradentes (UNIT) & \\
\hline \multirow{6}{*}{ Ceará (CE) } & \multirow{6}{*}{$\begin{array}{l}\text { Universidade Estadual } \\
\text { do Ceará (UECE) e } \\
\text { Universidade Federal } \\
\text { do Ceará (UFC*) }\end{array}$} & Universidade Federal do Ceará (UFC) & \multirow{6}{*}{$\begin{array}{l}\text { Profa. Dra. Selene Maia de } \\
\text { Morais - UECE e } \\
\text { Prof. Dr. Arlindo de Alencar } \\
\text { Araripe Moura - UFC }\end{array}$} \\
\hline & & Embrapa Agroindústria Tropical & \\
\hline & & Embrapa Caprinos & \\
\hline & & Universidade Estadual do Ceará (UECE) & \\
\hline & & Universidade de Fortaleza (UNIFOR) & \\
\hline & & Universidade Regional do Cariri (URCA) & \\
\hline \multirow{3}{*}{ Bahia (BA) } & \multirow{3}{*}{$\begin{array}{l}\text { Universidade Federal } \\
\text { da Bahia (UFBA) }\end{array}$} & Universidade Federal da Bahia (UFBA) & \multirow{3}{*}{$\begin{array}{l}\text { Prof. Dr. Paulo Fernando de } \\
\text { Almeida - UFBA }\end{array}$} \\
\hline & & Centro de Pesquisas Gonçalo Moniz (CPqGM) & \\
\hline & & Universidade Estadual de Santa Cruz (UESC) & \\
\hline \multirow{3}{*}{ Paraíba (PB) } & \multirow{3}{*}{$\begin{array}{l}\text { Universidade Federal } \\
\text { da Paraíba (UFPB) }\end{array}$} & Universidade Federal da Paraíba (UFPB) & \\
\hline & & Embrapa Algodão (CNPA) & $\begin{array}{l}\text { Prof. Dr. Demetrius Antônio } \\
\text { Machado de Araúio - UFPB }\end{array}$ \\
\hline & & Universidade Estadual da Paraíba (UEPB) & \\
\hline
\end{tabular}

* Não há Instituições ou Centros de Pesquisa Cadastrados como Associadas a esse Ponto Focal.

Quadro 1 - Representação das Instituições e Centros de Pesquisa Associadas aos Pontos Focais por Estado, como também os Respectivos Representantes Estaduais.

Fonte: Dados coletados na plataforma RENORBIO (Disponível em: <http://www.renorbio.org.br/portal/nucleode-posgraduacao/instituicoes.htm>. Acesso em: 9 dez. 2015). 
Observa-se a crescente incidência de institutos associados a partir dos pontos focais nos Estados de Pernambuco (sete institutos participantes) e Ceará (seis institutos participantes). Essa incidência é refletida sobre os pontos focais por serem os iniciantes na construção da Rede Nordeste de Biotecnologia (RENORBIO, [s.d.]e). Como forma de esclarecer melhor essa discussão, inferimos:

A proposta de integrar o Nordeste pela Biotecnologia passou por discussões que remontam ao ano de 1998. Entretanto, o Protocolo de Cooperação celebrado por todos os Secretários de C\&T dos Estados do NE, em agosto de 2003, em Fortaleza, hipotecando total apoio ao programa RENORBIO, foi a primeira medida de apoio formal que permitiu que o RENORBIO pudesse se estabelecer de modo a alcançar seus objetivos e garantir que os investimentos realizados atendessem aos padrões mais elevados de desempenho para todas as suas atividades. (RENORBIO, [s.d.]d).

Com isso, os Estados de Pernambuco e Ceará foram os pioneiros no recrutamento de incentivos fiscais e de pesquisa para o início da rede. Até porque a UFPE e a UECE foram algumas das poucas instituições que conseguiram aprovação em projetos iniciais de incentivo à criação da RENORBIO ([s.d.]c).

Percebe-se também que o Estado do Espírito Santo integra o quadro de pontos focais da RENORBIO, fato esse justificado pelo interesse e iniciativa dessa universidade pela integração junto à rede de biotecnologia (RENORBIO, [s.d.]c). Isso tornou possível uma rede de colaboração e produção científica e tecnológica da Biotecnologia no nordeste brasileiro.

Já o corpo docente da RENORBIO, por pontos focais, está representado pela figura 2, que mostra o número de docentes permanentes distribuídos pelos pontos focais.

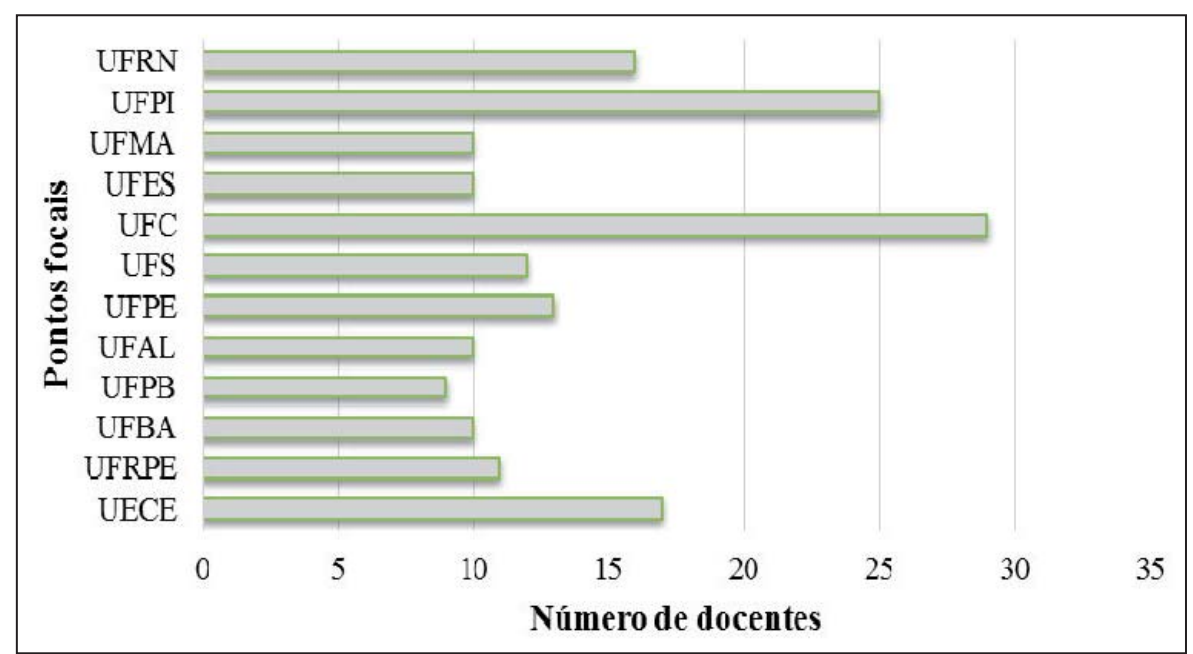

Figura 2 - Representação gráfica do número total de docentes permanentes, por pontos focais, do programa de pós-graduação da RENORBIO. Os dados foram expressos por números inteiros (RENORBIO, [s.d.]a).

Observa-se que há um total de 172 docentes permanentes, distribuindo-se em maior número nos pontos focais dos Estados do Ceará (29 docentes na UFC e 17 na UECE), do Piauí (25 docentes na UFPI) e do Rio Grande do Norte (16 docentes na UFRN).

\section{2 Áreas de concentração e doutorandos/tecendo o caminho ao título}

A RENORBIO está centralizada em quatro principais áreas de concentração, as quais possuem uma distribuição em diversas linhas de pesquisa, a saber: 
- Biotecnologia Industrial: voltada para produção de produtos via bioprocessos, como enzimas, biopolímeros, proteínas recombinantes, biossurfactantes e também na recuperação de bioprodutos e bioprocessos aplicados ao meio ambiente (COSTA, 2012 ; RENORBIO, [s.d.]h).

- Biotecnologia em Agropecuária: com as linhas de pesquisa voltadas para a sanidade animal, genética e transgênese e conservação e multiplicação de germoplasma; essa área de concentração debruça-se em técnicas diversas relacionadas com as linhas de pesquisa, como também em produtos formulados com o propósito de criopreservar células e tecidos, de produzir organismos geneticamente modificados e de diagnóstico e controle de doenças infecciosas aos animais (RENORBIO, [s.d.]h).

- Biotecnologia em Recursos Naturais: essa área de concentração envolve as linhas de pesquisa voltadas para a bioprospecção, biodiversidade e conservação, como também a purificação, caracterização e produção de insumos biotecnológicos (QUINTELLA et al., 2013; RENORBIO, [s.d.]h).

- Biotecnologia em Saúde: vacinas, hemoderivados, kits de diagnóstico, genômica, proteômica e nanobiotecnologia são as principais linhas de pesquisa que compõem essa área de concentração; tem como linhas de pesquisa o desenvolvimento de agentes profiláticos, terapêuticos e de diagnóstico (RENORBIO, [s.d.]h).

Além dessas áreas de concentração, a área de bionegócios e marcos legais (com as linhas de pesquisa bioética e marcos regulatórios e prospecção e transferência tecnológica), também faz parte do porte de escolha e de atuação durante o doutorado da RENORBIO (RENORBIO, [s.d.]h).

Nesse contingente, os discentes ingressantes ao programa têm a designação do seu projeto de doutoramento em uma das áreas de concentração, com as respectivas linhas de pesquisa.

Em relação ao número de ingressantes discentes, nos anos de 2006 a 2015, houve um maior número de ingressantes nos anos de 2014 (229 ingressantes discentes), 2015 (156 ingressantes discentes) e 2013 (146 ingressantes discentes) (Figura 3), deixando em evidência a crescente demanda de profissionais interessados pelo programa.

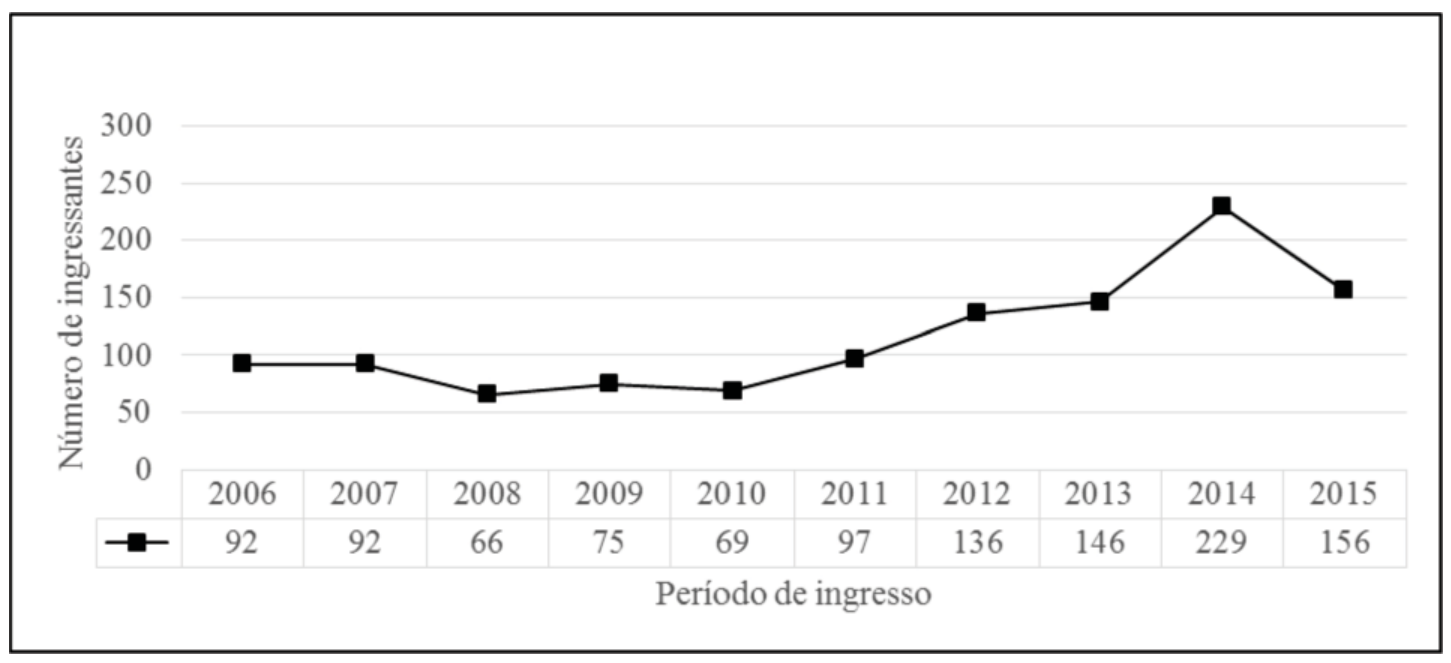

Figura 3 - Representação gráfica do número de discentes ingressantes nos anos de 2006 a 2015 no programa de pós-graduação da RENORBIO Fonte: Renorbio ([s.d.]j). 
Nos últimos 10 anos (2006 a 2015), a RENORBIO recebeu um número total de 1.158 alunos ingressantes, dos quais, 434 optaram pela área de concentração de biotecnologia da saúde, 352 em biotecnologia de recursos naturais, 201 em biotecnologia em agropecuária, 169 em biotecnologia industrial e, por fim, porém não menos importante, dois em bionegócios e marcos legais (Figura 4).

Percebe-se também que a área de concentração em biotecnologia em saúde foi a escoIhida pelos pesquisadores, haja vista que prevaleceu na maioria dos anos, seguida pelas áreas de biotecnologia de recursos naturais e agropecuária (Figura 4).

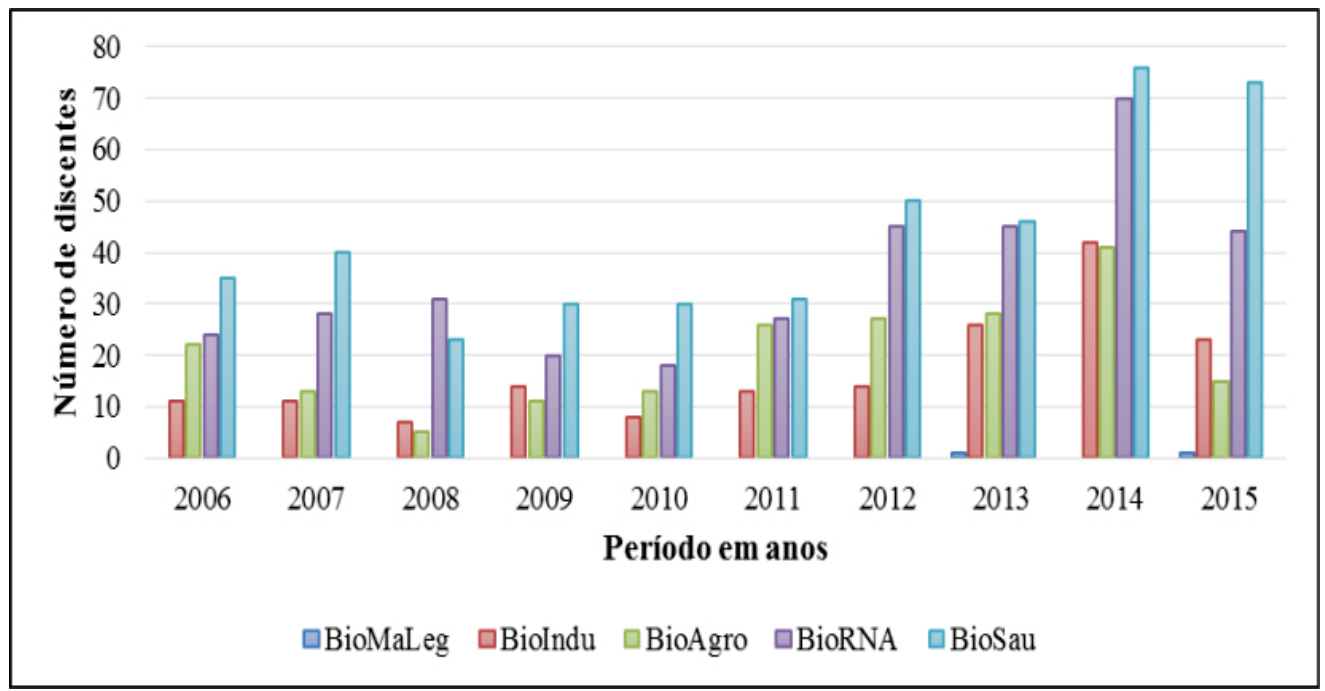

Figura 4 - Representação gráfica do número de discentes por área de concentração do programa de pós-graduação da RENORBIO no período de 2006 a 2015 Fonte: Renorbio ([s.d.]j).

\section{PRODUTOS E PROCESSOS BIOTECNOLÓGICOS}

A saber, em documento disponibilizado na plataforma digital da RENORBIO, há uma prevalência de 87 produtos e/ou processos formulados a partir dos trabalhos de doutoramento dos discentes do programa da RENORBIO (Tabela 1).

Tabela 1 - Quantificação de Produtos e Processos Formulados a partir de Teses do Programa da RENORBIO

\begin{tabular}{ccc}
\hline Área de aplicação & Número de produtos/processos & Pedido de patente \\
\hline Agricultura & 5 & 2 \\
Biofármacos & 29 & 24 \\
Bioindústria & 27 & 22 \\
Bioprospecção & 5 & 4 \\
Pecuária & 10 & 7 \\
Saúde & 11 & 8 \\
\hline Total: & 87 & 67
\end{tabular}

Fonte: Renorbio ([s.d.]f).

É notória a percentagem de produtos e processos nas mais diversas áreas de aplicação, levando em consideração o total de 67 (77\%) pedidos e/ou depósitos de patentes no Brasil e no exterior. 
Dessa forma, a RENORBIO destaca-se na produção de produtos formulados a partir dos trabalhos de doutoramento, resultando, por muitas vezes, em depósitos de patentes (COSTA, 2012; QUINTELLA et al., 2013).

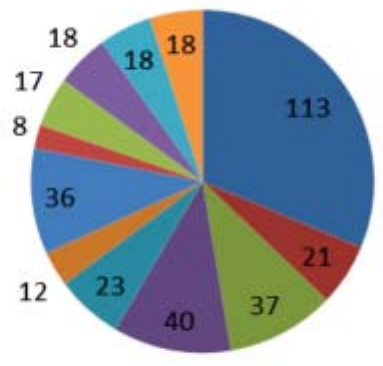

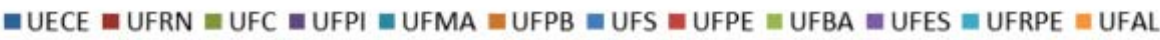

Figura 5 - Representação gráfica do total de teses defendidas do Programa de Pós-Graduação RENORBIO depositadas na Plataforma Sucupira no período de 2006 a 2015.

Fonte: Plataforma Sucupira (s.d.).

\section{O CONHECIMENTO DISPONÍVEL}

O total de teses depositadas na Plataforma Sucupira, pelo programa RENORBIO, no período de 2006 a 2015, equivale a 361 teses; a maior parte está interligada à UECE (31\%; 113 teses) (Figura 5). Já no banco de teses CAPES, observou-se um total de 163 teses, sendo que a UECE prevaleceu como a instituição de maior número de depósitos.

Em sequência, observa-se um total de 40 teses (11\%) defendidas pela UFPI, seguindo-se pela UFC (37 teses; 10\%) e pela UFS (36 teses; 10\%) (Figura 5).

Aqui é importante frisar que o tempo de existência que a instituição possui, que pode vir a ser um indicativo do seu desenvolvimento científico, não foi significativo nas instituições de maior idade. A saber, a UECE foi fundada em 1975 (40 anos), a UFPI, no ano de 1969 (47 anos), a UFC, em 1954 (61 anos) e a UFS, em 1963 (53 anos).

Em 2013, Quintella et al. relataram o desenvolvimento da produção científica oriunda das teses da RENORBIO. Sobre isso, os autores dizem:

Embora recente, a RENORBIO vem apresentando resultados bastante promissores, principalmente no que se refere à produção de conhecimento e geração de tecnologia, tanto na área de produtos quanto na área de processos. Segundo informações da RENORBIO, publicadas em março de 2012 no site do Centro de Inovação e Transferência de Tecnologia, a rede já formou 176 doutores e seus alunos produziram 146 criações que foram apropriadas como patentes. (QUINTELLA et al., 2013, p. 103).

Apesar de o programa RENORBIO ter formado a sua primeira turma de doutorandos no ano de 2006, foi apenas em 2008 que a CAPES integralizou a área de Biotecnologia como uma grande área de concentração, possibilitando, dessa forma, a criação de mais outros programas de pós-graduação em Biotecnologia (QUINTELLA et al., 2013).

Aqui é importante ressaltar a criação de novas redes em Biotecnologia, como a Rede Pró-Centro-Oeste (s.d.), formada no Centro-Oeste do País pelos Estados de Goiás, Mato Grosso 
do Sul, Mato Grosso, Distrito Federal e Paraná. Como também na Região Norte, com a Rede BIONORTE ; a qual abrange os Estados do Acre, Amazonas, Amapá, Pará, Maranhão, Rondônia, Roraima, Tocantins e Mato Grosso. Essas redes objetivam a formação e integração da PD\&I de profissionais em Biotecnologia e Biodiversidade nas respectivas regiões (BIONORTE, [s.d.]; REDE PRÓ-CENTRO-OESTE, s.d.) $)^{7}$.

Vale ressaltar que o número de teses defendidas pela RENORBIO nos anos de 2006 a 2015 foi considerado pelo total de teses depositadas na Plataforma Sucupira.

Até maio de 2011, um total de 576 artigos científicos e também pedidos de patentes foram acumulados através dos trabalhos de teses do programa RENORBIO (COSTA, 2012). Já no período de 2006 a 2011, foram contabilizados um total de 1.373 artigos científicos publicados por membros docentes ligados ao programa da RENORBIO (QUINTELLA et al., 2013), não havendo, necessariamente, uma relação direta com os discentes do programa.

Em uma busca avançada realizada no portal de periódicos da CAPES, foram detectados um total de 21 artigos científicos ligados ao programa RENORBIO, no período de 2006 a 2015.

No que se refere à BDTD, foi encontrado somente uma tese formulada pela RENORBIO e pela UFRN publicada em 2012, intitulada Da bancada ao Bureau: análise do desenvolvimento da biotecnologia no Nordeste Brasileiro sob o enfoque de Sistema Regional de Inovação, de autoria de Benedita Marta Gomes Costa e orientada pela Professora Doutora Gorete Ribeiro de Macedo da UFRN ${ }^{8}$ (BDTD, [s.d.]a).

Com o objetivo de reunir as teses e dissertações defendidas em todo o País e por brasileiros no exterior, a BDTD foi efetivada em abril de 2002 e, atualmente, possui um total de 251.106 dissertações e 93.587 teses (BDTD, [s.d.]b; [s.d.]c), servindo como fonte de pesquisa para metadados relacionados à diversidade de trabalhos formulados na pós-graduação do País.

\subsection{A biotecnologia como foco de pesquisa}

A Biotecnologia representa uma área promissora de aplicação industrial e consequentemente econômica. No entanto, por que essa área de conhecimento foi escolhida como foco de pesquisa? A RENORBIO explica:

O Brasil é um dos maiores detentores da biodiversidade. Já a Região Nordeste, possui $42 \%$ da sua área constituída pelo semi-árido; uma região com clima e biodiversidade únicos em todo o mundo. Essas e outras características tornam o cenário para a Biotecnologia muito promissor. O progresso nesta área poderá ser acelerado se houver esforços conjugados entre o governo, a comunidade científica e a empresarial no desenvolvimento de projetos conjuntos, na formação de parcerias produtivas, na capacitação de recursos humanos, na criação de ambiente favorável a novos investimentos e no desenvolvimento e/ou adaptação de tecnologias com o objetivo de ampliar a competitividade e dinamizar o mercado de produtos biotecnológicos. (RENORBIO, [s.d.]b.).

O diretório dos grupos de pesquisa do CNPq é formulado a partir das atividades dos grupos de pesquisa científica e tecnológica em exercício no País. Nesse sentido, esse diretório vem

\footnotetext{
${ }^{6}$ Programa de Pós-Graduação em Rede de Biodiversidade e Biotecnologia da Amazônia Legal.

${ }^{7}$ Apesar de alguns Estados não pertencerem, em termos federativos, à Região Norte e ao Centro-Oeste, eles agregam a Rede de Biotecnologia concernente aos presentes espaços territoriais.

${ }^{8}$ Para fins de esclarecimento, destaca-se que o valor de um trabalho encontrado na BDTD divergente ao total de 361 trabalhos encontrados na Plataforma Sucupira, pode ser explicado, provavelmente, pelo fato do não repasse/ depósito dos estudos no referido banco de dados.
} 
capacitando a descrição do perfil generalizado das principais atividades envolvidas desses grupos de pesquisa (CNPQ, [s.d.]a.).

Dessa forma, a partir de pesquisas realizadas pelo diretório de pesquisa do CNPq, verificou-se a existência de 88 grupos de pesquisa no Nordeste que usam o termo Biotecnologia no nome desses grupos. Desse total, $46 \%$, ou seja, 41 grupos são pertencentes às instituições de ensino superior que são pontos focais da RENORBIO (CNPQ, [s.d.]b).

As universidades UECE, UFC, UFPE, UFPB e a UFBA, foram as de maior destaque no número de grupos de pesquisa cadastrados no diretório de pesquisa do CNPq.

Esses dados mostram o quanto a biotecnologia vem crescendo em relação ao campo de pesquisa científica e tecnológica no País e, principalmente, no nordeste brasileiro, representando uma grande parcela de produção científica para o Brasil.

\section{CONSIDERAÇÕES FINAIS}

A RENORBIO, uma iniciativa governamental que tem como objetivo medular alavancar profissionais na área de Biotecnologia nos setores de ensino e pesquisa, contribuindo fielmente com o desenvolvimento da biotecnologia no nordeste brasileiro, é um programa que vem se concretizando no cenário científico.

Vê-se o quanto do crescimento e das contribuições que o programa de pós-graduação da RENORBIO alcançou no período de 2006, ano de sua criação, até o ano de 2015, ano deste estudo, recebendo notório respeito na formação de 361 doutores em biotecnologia e de mais de 140 depósitos de patentes, aptos a se debruçarem no desenvolvimento científico e tecnológico do País, como também na formação de novos profissionais de nível superior na pós-graduação.

A partir desse cenário, tem-se como suporte que a Biotecnologia é uma grande área de conhecimento que proporciona estudos promissores com a finalidade de se obter produtos, processos e serviços, os quais auxiliam no desenvolvimento da ciência, tecnologia e inovação no âmbito nacional e internacional.

\section{REFERÊNCIAS}

BIBLIOTECA DIGITAL BRASILEIRA DE TESES E DISSERTAÇÕES (BDTD). Busca Avançada. Brasília: Instituto Brasileiro de Informação em Ciência e Tecnologia, [s.d.]a. Disponível em: <http://bdtd.ibict.br/vufind/ Search/Advanced>. Acesso em: 11 jan. 2016.

. O que é? Brasília: Instituto Brasileiro de Informação em Ciência e Tecnologia, [s.d]b. Disponível em: <http://bdtd.ibict.br/vufind/Content/whatls>. Acesso em: 11 jan. 2016.

. Número de documentos por tipo de documento. Brasília: Instituto Brasileiro de Informação em Ciência e Tecnologia, [s.d.]c. Disponível em: <http://bdtd.ibict.br/vufind/Statistics/Count?field=format>. Acesso em: 11 jan. 2016.

BRASIL. Regimento do Programa de Pós-Graduação Stricto Sensu em Biotecnologia da Renorbio. Brasília: Ministério da Ciência e Tecnologia, [s.d.]. Disponível em: <http://www.renorbio.org.br/portal/documentos/ regimento-do-curso.htm. 2015>. Acesso em: 15 dez. 2015.

COORDENAÇÃO DE APERFEIÇOAMENTO DE PESSOAL DE NÍVEL SUPERIOR (CAPES). Banco de Teses CAPES. Brasília: Ministério da Educação, [s.d.]. Disponível em: <http://bancodeteses.capes.gov.br/>. Acesso em: 11 jan. 2016.

COSTA, Benedita. Da bancada ao bureau: análise do desenvolvimento da biotecnologia no Nordeste brasileiro sob o enfoque do sistema regional de inovação. 2012. 234f. Tese (Doutorado em Biotecnologia) 
- Universidade Federal do Rio Grande do Norte (UFRN) e Rede Nordeste de Biotecnologia (RENORBIO), Natal, RN, 2012.

CONSELHO NACIONAL DE DESENVOLVIMENTO CIENTÍFICO E TECNOLÓGICO (CNPq). O diretório: o que é. Brasília: Ministério da Educação, [s.d.]a. Disponível em: <http://lattes.cnpq.br/web/dgp/o-que-e/>. Acesso em: 12 jan. 2016.

. Consulta parametrizada. Brasília: Ministério da Educação, [s.d.]b. Disponível em: <http://dgp. cnpq.br/dgp/faces/consulta/consulta_parametrizada.jsf>. Acesso em: 12 jan. 2016.

GIL, Antônio Carlos. Métodos e técnicas de pesquisa social. 6. ed. São Paulo: Atlas, 2008.

PLATAFORMA SUCUPIRA. Trabalho de conclusão: dados para consulta. Brasília: Capes, [s.d.]. Disponível em: <https://sucupira.capes.gov.br/sucupira/public/consultas/coleta/trabalhoConclusao/ listaTrabalhoConclusao.jsf>. Acesso em: 30 maio 2016.

QUINTELLA, C. M. et al. Política de Estado de Inovação Tecnológica: a Renorbio na biotecnologia do nordeste do Brasil. In: RUSSO, Suzana Leitão; SILVA, Gabriel Francisco da (Org.). Capacite: exemplos de inovação tecnológica. São Cristóvão, SE: Editora UFS, 2013. 240p.

REDE DE BIODIVERSIDADE E BIOTECNOLOGIA DA AMAZÔNIA LEGAL (BIONORTE). Manaus: Ministério da Ciência, Tecnologia e Inovação, [s.d.]. Disponível em: <http://www.bionorte.org.br/bionorte.htm>. Acesso em: 30 maio 2016.

REDE NORDESTE DE BIOTECNOLOGIA (RENORBIO). Renorbio: sobre a Rede Nordeste de Biotecnologia. Recife, [s.d.]a. Disponível em: <http://www.renorbio.org.br/portal/renorbio.htm>. Acesso em: 2 nov. 2015.

Biotecnologia: conheça um pouco mais sobre a biotecnologia. Recife, [s.d.]b. Disponível em: <http://www.renorbio.org.br/portal/renorbio/biotecnologia.htm>. Acesso em: 2 nov. 2015.

Núcleo de pós-graduação: conheça um pouco mais sobre o programa de pós-graduação do Renorbio. Recife, [s.d.]c. Disponível em: <http://www.renorbio.org.br/portal/nucleo-de-posgraduacao. htm>. Acesso em: 11 nov. 2015.

. Histórico: conheça melhor a história do Renorbio. Recife, [s.d.]d. Disponível em: <http://www. renorbio.org.br/portal/renorbio/historico.htm>. Acesso em: 5 dez. 2015.

. Pontos focais: conheça os pontos focais do núcleo de pós-graduação. Recife, [s.d.]e. Disponível em: <http://www.renorbio.org.br/portal/nucleo-de-posgraduacao/pontos-focais.htm>. Acesso em: 9 dez. 2015.

Biotecnologias: conheça os bioprodutos e bioprocessos desenvolvidos no âmbito da Renorbio. Recife, [s.d.]f. Disponível em: <http://www.renorbio.org.br/portal/biotecnologias.htm>. Acesso em: 30 nov. 2015.

Infra-estrutura: conheça os laboratórios disponíveis para a Renorbio. Recife, [s.d.]g. Disponível em: <http://www.renorbio.org.br/portal/nucleo-de-posgraduacao/infraestrutura.htm>. Acesso em: 5 jan. 2016.

Áreas de concentração: o Renorbio possui quatro áreas de concentração conforme o quadro abaixo. Recife, [s.d.]h. Disponível em: <http://www.renorbio.org.br/portal/nucleo-de-posgraduacao/ areas-de-concentracao.htm>. Acesso em: 5 jan. 2016.

. Destaques: destaques do Renorbio. Recife, [s.d.]i. Disponível em: <http://www.renorbio.org.br/ portal/destaques.htm?act=view\&pgn=1>. Acesso em: 23 jan. 2016.

Corpo discente: alunos do Núcleo de Pós-Graduação da RENORBIO. Recife, [s.d.]j. Disponível em: <http://www.renorbio.org.br/portal/nucleo-de-posgraduacao/corpo-discente.htm>. Acesso em: 7 jan. 2016.

REDE PRÓ-CENTRO-OESTE. Quem somos? Rede Pro Centro-Oeste. Brasília: Ministério da Ciência, Tecnologia e Inovação, [s.d.]. Disponível em: <http://200.129.206.69/redepro/index/i/9/quem-somos>. Acesso em: 30 maio 2016. 


\section{Sobre os autores:}

Mário Luan Silva de Medeiros: Doutorando e Mestre em Bioquímica e Biologia Molecular pela Universidade do Estado do Rio Grande do Norte (UERN), Especialista em Biotecnologia pela Universidade Católica Dom Bosco (UCDB) e Bacharel em Biotecnologia pela Universidade Federal Rural do Semi-Árido (UFERSA). Professor na UERN. E-mail: marioluan@oi.com.br

Josimara Nolasco Rondon: Pós-Doutora pela Universidade Católica Dom Bosco (UCDB). Mestrado e Doutorado em Biologia Vegetal pela Universidade Estadual de Campinas (UNICAMP). Graduação em Ciências Biológicas pela Universidade Federal do Mato Grosso do Sul (UFMS). Professora em Biologia Vegetal na UCDB. E-mail: 4132@ucdb.br 
